\title{
Polarized interacting exciton gas in quantum wells and bulk semiconductors
}

\author{
J. Fernández-Rossier and C. Tejedor \\ Departamento de Física Teórica de la Materia Condensada, Universidad Autónoma de Madrid, Cantoblanco, 28049 Madrid, Spain \\ L. Muñoz and L. Vĩ̃a \\ Departamento de Física de Materiales, Universidad Autónoma de Madrid, Cantoblanco, 28049 Madrid, Spain
}

(Received 26 September 1995; revised manuscript received 22 January 1996)

\begin{abstract}
We develop a theory to calculate exciton binding energies of both two- and three-dimensional spin polarized exciton gases within a mean field approach. Our method allows the analysis of recent experiments showing the importance of the polarization and intensity of the excitation light on the exciton luminescence of GaAs quantum wells. We study the breaking of the spin degeneracy observed at high exciton density $\left(5 \times 10^{10}\right.$ $\mathrm{cm}^{2}$ ). Energy level splitting between spin +1 and spin -1 is shown to be due to many-body interexcitonic exchange while the spin relaxation time is controlled by intraexciton exchange. [S0163-1829(96)06224-8]
\end{abstract}

\section{INTRODUCTION}

The optical response of intrinsic semiconductor heterostructures has received considerable attention in recent years from both the theoretical and the experimental point of view. The study of the luminescence spectrum gives information about the lowest electronic excited state of a semiconductor, i.e., the exciton. Using polarized light, emitted photons contain information on both exciton energies and their dependence on the exciton spin. ${ }^{1}$ Time resolved photoluminescence experiments ${ }^{2-6}$ provide information on different exciton properties: exciton formation and decay processes, spin relaxation, binding energy evolution, etc. In some of those experiments, performed in the picosecond range, ${ }^{2-6}$ an energy splitting between spin +1 and spin -1 excitons has been reported. These studies show that the spin splitting increases with both the initial exciton density and the degree of initial polarization.

So far, theoretical models have been proposed ${ }^{7,8}$ to explain only the exciton spin relaxation without taking into account the exciton-exciton interaction. The models give alternative explanations to spin relaxation processes in terms of intraexcitonic exchange, ${ }^{9-12}$ D'yakonov-Perel, ${ }^{13}$ and Elliot-Yaffet ${ }^{14}$ mechanisms. However, these free-exciton models fail to describe the spin level splitting.

As far as many-exciton effects are concerned, only the spinless high density exciton gas has been the subject of theoretical research in the last 30 years. ${ }^{15-18}$ Three different schemes have been developed. The first one, proposed by Keldysh $^{18}$ and generalized by Comte and Nozieres, ${ }^{16}$ is a BCS-like approach. Another method, due to Hanamura and Haug, ${ }^{15}$ consists in writing the Hamiltonian in terms of exciton operators. When the center of mass momentum of each exciton is zero these two approaches are equivalent up to order $\left(n a^{d}\right)^{2}$, where $n$ is the exciton densty, $a$ is the exciton radius $(\hbar)^{2} \epsilon / \mu e^{2}, e$ being the electron charge, $\mu$ the reduced exciton mass, and $\epsilon$ the dielectric constant, and $d$ is the dimension of the space. The last availaible theoretical scheme consists in writing a Bethe-Salpeter equation and interpreting the homogeneous part as a multiexciton Wannier equation. ${ }^{19,20}$ The physics underlying the three approaches is always a mean field treatment of interaction between spinless excitons and so the equations obtained are analogous. The differences lie in the obtaining of the equations and in the physical nature of the mathematical objects the theories are built upon. In any case, spin splitting is beyond the scope of those spinless excitons theories.

We present in this paper a theory of spin-dependent exciton-exciton interaction in two and three dimensions (2D and 3D). Such interaction produces a gas with a difference in the spin populations, a level splitting. We concentrate on the spin and energy dynamics and their effect on the exciton recombination without paying attention to the exciton formation. Our theory for spin polarized systems is an extension of the exciton operator Hamiltonian approach (Hanamura-Haug approach $\left.^{15}\right)$. Our results for the 2D case are in agreement with experiments in quantum wells. ${ }^{2-6}$ Experimental work in the interacting regime with polarized light remains to be done in bulk, to the best of our knowledge.

This paper is organized as follows. In Sec. II we present the many-exciton Hamiltonian ${ }^{17}$ from which we obtain many-exciton Wannier equations including spin. In Sec. III we solve them using both perturbative and variational approaches. The main approximation we make is to consider the exciton center of mass at rest. Our calculations, correct up to order $\left(n a^{d}\right)^{2}$, give four exciton energies and wave functions as a function of density in two and three dimensions. The maximum density experimentally reached is about $10^{11}$ excitons $\mathrm{cm}^{-2}$. A reasonable estimate for the exciton radius is $100 \AA$. When $n=10^{11} \mathrm{~cm}^{-2}$ then $n a^{d}$ is roughly 0.1 . This means we neglect $10^{-2}$ compared with $10^{-1}$. Screening corrections to the energy within RPA are also taken into account although they do not depend on the exciton spin. A rate equation is proposed and solved in Sec. IV in order to obtain the time evolution of the different types of excitons. Once we have the energies as a function of densities and the densities as a function of time, we can get results directly comparable with experiments obtaining a qualitatively good agreement. Finally, Sec. V is devoted to the discussion and summary of our main results. 


\section{INTERACTING POLARIZED EXCITON GAS THEORY}

Dealing with exciton-exciton interaction is a complicated task. The exciton is a two-body excitation which is not, properly speaking, a boson. Even the noninteracting exciton Green function is not simple to calculate. ${ }^{21-23}$ Consider the exciton creation operator:

$$
\psi_{i}^{\dagger}=\int \operatorname{dedh} \phi_{i}(e, h) \psi_{h}^{\dagger} \psi_{e}^{\dagger},
$$

where the index $i$ is the set of exciton quantum numbers $\{\mathbf{K}, J, M, \nu\}, \mathbf{K}$ being the center of mass momentum, $J$ the total angular momentum, $M$ its third component, and $\nu$ labeling the internal state of the exciton. $\psi_{e}^{\dagger}$ creates a conduction band electron in $e$, where $e=\left\{\mathbf{r}, s_{e}\right\}$, and analagously $\psi_{h}^{\dagger}$ for a hole for which we neglect valence band mixing effects. On the other hand, $\phi_{i}(e, h)$ is the exciton wave function. So, physically speaking, $\psi_{i}^{\dagger}$ is an operator that creates an electron-hole cloud following the excitonic probability amplitude. The exciton wave function can be written as a product of factors, namely, the center of mass term, the or- bital part, and the radial wave function. This factorization is possible if we assume that $\{\mathbf{K}, J, M, \nu\}$ are good quantum numbers for the noninteracting exciton.

Up to order $\left(n a^{d}\right)^{2}$ it can be shown ${ }^{17}$ that the exciton operator Hamiltonian including the exciton exciton interaction can be written as

$$
H=H_{0}+H_{\text {int }},
$$

where

$$
H_{0}=\sum_{i, i^{\prime}}\left\langle i\left|T_{e}+T_{h}-V_{e h}\right| i^{\prime}\right\rangle \psi_{i}^{\dagger} \psi_{i^{\prime}}
$$

and

$$
\begin{aligned}
H_{\text {int }}= & \frac{1}{2} \sum_{i, i^{\prime}, i^{\prime \prime}, i^{\prime \prime \prime}} \psi_{i}^{\dagger} \psi_{i^{\prime}}^{\dagger} \psi_{i^{\prime \prime}} \psi_{i^{\prime \prime \prime}}\left(\left\langle i, i^{\prime}\left|I_{d}\right| i^{\prime \prime}, i^{\prime \prime \prime}\right\rangle\right. \\
& \left.+\left\langle i, i^{\prime}\left|I_{x}\right| i^{\prime \prime}, i^{\prime \prime \prime}\right\rangle\right),
\end{aligned}
$$

where

$$
\begin{gathered}
\left\langle i\left|T_{e}+T_{h}-V_{e h}\right| i^{\prime}\right\rangle=\int d e d h \phi_{i}^{*}(e, h)\left(T_{e}+T_{h}-V_{e h}\right) \phi_{i^{\prime}}(e, h), \\
\left\langle i, i^{\prime}\left|I_{d}\right| i^{\prime \prime}, i^{\prime \prime \prime}\right\rangle=\int d e d h d e^{\prime} d h^{\prime} \phi_{i}^{*}(e, h) \phi_{i^{\prime}}^{*}\left(e^{\prime}, h^{\prime}\right)\left(V_{e e^{\prime}}+V_{h h^{\prime}}-V_{e h^{\prime}}-V_{h e^{\prime}}\right) \phi_{i^{\prime \prime}}(e, h) \phi_{i^{\prime \prime \prime}}\left(e^{\prime}, h^{\prime}\right), \\
\left\langle i, i^{\prime}\left|I_{x}\right| i^{\prime \prime}, i^{\prime \prime \prime}\right\rangle=-\int d e d h d e^{\prime} d h^{\prime} \phi_{i}^{*}(e, h) \phi_{i^{\prime}}^{*}\left(e^{\prime}, h^{\prime}\right)\left(V_{e e^{\prime}}+V_{h h^{\prime}}-V_{e h^{\prime}}-V_{h e^{\prime}}\right) \phi_{i^{\prime \prime}}\left(e^{\prime}, h\right) \phi_{i^{\prime \prime \prime}}\left(e, h^{\prime}\right) .
\end{gathered}
$$

It must be stressed that this Hamiltonian is correct up to order $\left(n a^{d}\right)^{2}$. This means that using this Hamiltonian we can (and we must) neglect the contributions to the energy of order $\left(n a^{d}\right)^{2}$. The physical origin of $I_{d}$ is the direct unscreened Coulomb interaction between fermions belonging to different excitons while $I_{x}$ is the interexcitonic exchange, or the unscreened exchange interaction between fermions of the same type. It is important to distingish between interexcitonic exchange and intraexcitonic exchange. The former is, as we stated before, a many exciton intraband (conductionconduction or valence-valence) exchange and the latter is a single exciton or interband (conduction-valence) effect. ${ }^{9-11}$ The intraexcitonic exchange does not break the symmetry between spin +1 and spin -1 excitons and has a very weak influence in the exciton energy levels. ${ }^{7}$ In this paper we neglect the intraexcitonic exchange in the calculation of the exciton binding-energy. Nevertheless, the intraexcitonic exchange plays a very important role in the spin flip mechanism. ${ }^{7,8}$

We use a mean field approximation. First, we calculate the expectation value of the Hamiltonian with a wave function equal to the product of the noninteracting exciton wave functions:

$$
\begin{aligned}
\langle H\rangle= & \sum_{i} \omega_{i}^{0} n_{i}+\frac{1}{2} \sum_{i, i^{\prime}}\left(\left\langle i^{\prime}, i\left|I_{d}+I_{x}\right| i^{\prime}, i\right\rangle\right. \\
& \left.+\left\langle i^{\prime}, i\left|I_{d}+I_{x}\right| i, i^{\prime}\right\rangle\right) n_{i^{\prime}} n_{i}
\end{aligned}
$$

with

$$
\omega_{i}^{0}=\left\langle i\left|T_{e}+T_{h}-V_{e h}\right| i\right\rangle .
$$

Now, if we make a functional derivation without any further assumption about the single-exciton wave function, the Euler-Lagrange equations we obtain are terribly complicated. ${ }^{15,17,24}$ One of the complications is nonlocality, another is our lack of knowledge of $n_{i}$, the quantum nonequilibrium distributions. If we assume $\mathbf{K}=\mathbf{0}$, say, all the excitons are at rest, nonlocality disappears, and instead of a set of continuous functions $n(\mathbf{K}, J, M, \nu)$ we have a set of discrete numbers $n(J, M, \nu)$. This is the most drastic assumption we make. In the case of resonant excitation, the $\mathbf{K}=\mathbf{0}$ hypothesis is more realistic than in the nonresonant excitation case because, in the resonant regime, the system receives just the energy required to create the exciton without any kinetic energy. Experimental information is available both in the resonant ${ }^{2-5}$ and in the nonresonant ${ }^{2,3}$ regime. We 
consider only the resonant case, i.e., $\mathbf{K}=\mathbf{0}$. A less drastic and more usual assumption is that all the excitons are in their ground state $\nu=1$. So, spin $M$ is the only quantum number labeling our excitons. Since holes at the top of the valence band have an angular momentum $3 / 2$ and electrons a $1 / 2$ one, excitons have angular momenta running from 2 to -2 . Zero angular momentum does not play any role in the usual experimental configuration of light propagation along the growth axis in 2D systems. Hence, in order to describe the system we have only to know four numbers, i.e., the exciton populations which we denote by the array $\mathbf{n} \equiv\left\{n_{+2}, n_{+1}, n_{-1}, n_{-2}\right\}=\left\{n_{+2}, n_{\uparrow}, n_{\downarrow}, n_{-2}\right\}$. Another approximation implicit to the derivation of the Euler-Lagrange equations is that for each $\mathbf{n}$ the energy takes its equilibrium value. This is equivalent to saying that the changes in $\mathbf{n}$ are long compared with collision exciton times: the excitons interact between themselves many times before the populations change. This approximation is usually called adiabatic or quasiequilibrium. Therefore, $\mathbf{n}$ can be taken as fixed in the theory to compute energy levels.

Before obtaining the Euler-Lagrange equations, let us discuss the shape of the Hamiltonian. Substituting $i$ by $M$ in Eq. (6), $\langle H\rangle$ is a sum of integrals, but all the terms $\left\langle I_{d}\right\rangle$ are zero. This is a nice consequence of the approximation $\mathbf{K}=\mathbf{0}$ and the neutral charge of the exciton. We factorize the expectation values $\left\langle I_{x}\right\rangle$ in spin part and spatial part. Let us first discuss the former and second the latter. The spin part depends on the dimensionality of the space. We shall use the following notation: $\xi_{s_{e}, s_{h}}(M)$ for the probability amplitude of the electron having spin $s_{e}$ and hole having spin $s_{h}$ in an exciton with spin $M$. The spin part of $\left\langle I_{x}\right\rangle$ reads

$$
\begin{aligned}
I_{M_{1}, M_{2}}^{M_{3}, M_{4}}= & \sum_{s_{e}, s_{h}, s_{e}^{\prime}, s_{h}^{\prime}} \xi_{s_{e}, s_{h}}^{*}\left(M_{1}\right) \xi_{s_{e}^{\prime}, s_{h}^{\prime}}^{*}\left(M_{2}\right) \xi_{s_{e}^{\prime}, s_{h}}\left(M_{3}\right) \\
& \times \xi_{s_{e}, s_{h}^{\prime}}\left(M_{4}\right) .
\end{aligned}
$$

Obviously the spin wave functions depend on the confinement of the excitons. In the ideal 2D case, we consider the excitons as built up only from heavy holes. This is only a qualitative approximation for analyzing actual quantum wells where strong mixing effects can appear in optical properties. ${ }^{25,26}$ When just heavy holes are involved, the only possibility for the probability amplitudes is to take the form

$$
\begin{gathered}
\xi_{s_{e}, s_{h}}(+2)=\delta_{s_{e}, 1 / 2} \delta_{s_{h}, 3 / 2}, \xi_{s_{e}, s_{h}}(+1)=\delta_{s_{e},-1 / 2} \delta_{s_{h}, 3 / 2} \\
\xi_{s_{e}, s_{h}}(-1)=\delta_{s_{e}, 1 / 2} \delta_{s_{h},-3 / 2} \\
\xi_{s_{e}, s_{h}}(-2)=\delta_{s_{e},-1 / 2} \delta_{s_{h},-3 / 2}
\end{gathered}
$$

For the bulk case $\xi_{s_{e}, s_{h}}(M)$ is set equal to the ClebschGordan coefficient with $J=2$ in analogy with the 2D case in which third components of the angular momentum equal to \pm 2 appear. If the actual 3D exciton wave function should be a combination of $J=2$ and $J=1$, some quantitative changes would appear although the final results would remain qualitatively unaltered. Using Eq. (9), the only nonzero spin terms for the $2 \mathrm{D}$ case are

$$
A_{2 \mathrm{D}} \equiv I_{1,1}^{1,1}=1, \quad I_{1,2}^{2,1}=I_{1,-2}^{1,-2}=I_{2,1}^{1,2}=I_{-2,1}^{-2,1}=1,
$$

as well as the ones generated by the following symmetry properties:

$$
\begin{gathered}
I_{\alpha, \beta}^{\gamma, \delta}=I_{-\alpha,-\beta}^{-\gamma,-\delta}, \\
I_{\alpha, \beta}^{\gamma, \delta}=I_{\beta, \alpha}^{\delta, \gamma} .
\end{gathered}
$$

In the $3 \mathrm{D}$ case the only nonzero spin terms are

$$
\begin{array}{lll}
I_{2,2}^{2,2}=1, & A_{3 \mathrm{D}} \equiv I_{1,1}^{1,1}=\frac{10}{16}, & I_{1,-1}^{1,-1}=\frac{6}{16}, \\
I_{2,1}^{2,1}=\frac{3}{4}, & I_{2,1}^{1,2}=\frac{1}{4}, & I_{2,-1}^{2,-1}=\frac{1}{4},
\end{array}
$$

and the ones generated by Eq. (11). The main difference between Eqs. (10) and (12) is that $f_{d} \equiv I_{-1,1}^{-1,1}$ is not zero in the $3 \mathrm{D}$ case. These spin terms are proportional to the interaction term $n_{M} n_{-M}$ in Eq. (6). The physical consequence of this fact is that, in 2D, $M$ excitons do not exchange with $(-M)$ excitons and this enhances the splitting when $n(M)$ $\neq n(-M)$. As it will become clear in Sec. III, if we had $I_{-1,1}^{-1,1}=I_{1,1}^{1,1}$ then the splitting would be zero. In order to compare with the experimental results performed in quantum wells, we shall use our 2D results including a renormalized " $f_{d}$ term" $\left(I_{-1,1}^{-1,1}\right)$ interpolated between the $2 \mathrm{D}$ and the 3D values ( 0 and $3 / 8$, respectively) as well as a noninteracting exciton binding energy $E_{0}^{\mathrm{QW}}=E_{0}^{2 \mathrm{D}} / 2=2 E_{0}^{3 \mathrm{D}} \cdot \cdot^{27,28}$

In order to work out the spatial part, we proceed to make the functional derivation: $\left[\delta\langle H\rangle / \delta \phi_{M}(r)\right] / n(M)=0$ to get four Euler-Lagrange equations, one for each $M$. In these four equations there are two well differentiated parts, one corresponding to $\delta\left\langle H_{0}\right\rangle / \delta \phi_{M}(r)$ and the other corresponding to $\delta\left\langle H_{\text {int }}\right\rangle / \delta \phi_{M}(r)$. The former generates the usual Wannier equation ${ }^{17}$ and the latter generates the interaction terms. In order to reduce the four interaction terms $V\left(e, e^{\prime}\right), V\left(h^{\prime}, h\right), V\left(e, h^{\prime}\right), V\left(e^{\prime}, h\right)$ to one, i.e., $V(q)$, it is convenient to work in the momentum representation. We are going first to derive the equations in the case $n(2)=n(-2)=0$. This will simplify considerably the equations and will shed some light on the underlying physics. Besides, in the experiments the \pm 2 optically inactive ${ }^{7}$ excitons are less populated than the optically active \pm 1 . Hence, we have only two kinds of excitons, namely, up and down, $\uparrow$ and $\downarrow$. We omit the algebra because it does not give any physical information. The two multiexciton polarized Wannier equations are (we set $\hbar=1$ )

$$
\begin{aligned}
{\left[\frac{q^{2}}{2 \mu}-\right.} & \left.E_{\uparrow}\right] \phi_{\uparrow}(q)-\gamma_{\uparrow}(q)+2 A_{d} n_{\uparrow}\left[\left|\phi_{\uparrow}(q)\right|^{2} \gamma_{\uparrow}(q)\right. \\
& \left.-\Sigma_{\uparrow \uparrow}(q) \phi_{\uparrow}(q)\right]+2 f_{d} n_{\downarrow}\left[\gamma_{\downarrow}(q) \phi_{\downarrow}^{*}(q) \phi_{\uparrow}(q)\right. \\
& \left.+\left|\phi_{\downarrow}(q)\right|^{2} \gamma_{\uparrow}-\Sigma_{\downarrow \downarrow}(q) \phi_{\uparrow}(q)-\Sigma_{\downarrow \uparrow}(q) \phi_{\downarrow}(q)\right]=0,
\end{aligned}
$$




$$
\begin{aligned}
{\left[\frac{q^{2}}{2 \mu}\right.} & \left.-E_{\downarrow}\right] \phi_{\downarrow}(q)-\gamma_{\downarrow}(q)+2 A_{d} n_{\downarrow}\left[\left|\phi_{\downarrow}(q)\right|^{2} \gamma_{\downarrow}(q)\right. \\
& \left.-\Sigma_{\downarrow \downarrow} \phi_{\downarrow}(q)\right]+2 f_{d} n_{\uparrow}\left[\gamma_{\uparrow}(q) \phi_{\uparrow}^{*}(q) \phi_{\downarrow}(q)+\left|\phi_{\uparrow}(q)\right|^{2} \gamma_{\downarrow}\right. \\
& \left.-\Sigma_{\uparrow \uparrow}(q) \phi_{\downarrow}(q)-\Sigma_{\uparrow \downarrow}(q) \phi_{\uparrow}(q)\right]=0,
\end{aligned}
$$

with

$$
\begin{gathered}
\gamma_{s}(q) \equiv \int \frac{d \vec{t}}{(2 \pi)^{d}} \phi_{s}(\vec{t}+\vec{q}) V(\vec{t}), \\
\Sigma_{s, s^{\prime}}(q) \equiv \int \frac{d \vec{t}}{(2 \pi)^{d}} \phi_{s}^{*}(\vec{t}+\vec{q}) \phi_{s^{\prime}}(\vec{t}+\vec{q}) V(\vec{t}),
\end{gathered}
$$

where $V(\vec{s})$ is the Fourier-transform of the bare Coulomb potential and $E_{\uparrow}$ and $E_{\downarrow}$ are the Lagrange multipliers associated to the normalization constraint which are interpreted as the exciton energies. Note that Eqs. (13), (14), and (15) depend on the dimension through $\phi, A_{d}$, and $f_{d}$. The first two terms in Eqs. (13) and (14) are the usual Wannier interactionless, two band, exciton equation. The other terms proportional to $n$ and $n_{\uparrow}$ represent the mean field exciton-exciton interaction. Observe that we can obtain each equation by reversing all the spins of the other because there is no magnetic field. The spin symmetry breaking comes from the fact that $n_{M} \neq n_{-M}$.

\section{CALCULATION OF THE INTERACTING EXCITON ENERGY LEVELS}

\section{A. Perturbation theory}

Solving the multiexciton Wannier equations (13) and (14) exactly is not possible and therefore we use some approximations. First we calculate $E$ perturbatively, using two first terms in Eqs. (13) and (14) as an unperturbed Hamiltonian and the interacting terms as the perturbation. The perturbation parameter is obviously $n a^{d}$ and, as we stressed before, we must drop all the contributions to the energy with order higher than $n a^{d}$. Consequently, we do not go further than first order perturbation theory, which means that the interacting exciton wave function is the same as the noninteracting one. Hence, to first order in perturbation theory, $\phi_{\downarrow}=\phi_{\uparrow}=\phi_{0}$ where

$$
\phi_{0}^{2 \mathrm{D}}(q)=\frac{(2 \pi)^{1 / 2} a}{\left[1+(a q / 2)^{2}\right]^{3 / 2}}, \quad \phi_{0}^{3 \mathrm{D}}(q)=\frac{8\left(\pi a^{3}\right)^{1 / 2}}{\left[1+(a q)^{2}\right]^{2}},
$$

are the exciton wave functions in the isotropic, parabolic two band model. ${ }^{29}$ In the momentum representation the Schrödinger equation is an integral equation. ${ }^{30}$ The perturbation gives a first order correction

$$
\begin{aligned}
\Delta E= & \int \frac{d \vec{q}}{(2 \pi)^{d}}\left|\phi_{0}(q)\right|^{2} \Delta H(q) \\
& +\int \frac{d \vec{q}}{(2 \pi)^{d}} \phi_{0}(q) \int \frac{d \vec{t}}{(2 \pi)^{d}} \Delta H(\vec{t}, \vec{p}) \phi_{0}(\vec{t}+\vec{p}) .
\end{aligned}
$$

Using Eq. (13) in Eq. (17) we obtain the following expression for the $2 \mathrm{D}$ case:

$$
\begin{aligned}
& \Delta E_{\uparrow}=2 n_{\uparrow}\left(I_{1}-I_{2}\right)+2 f_{\mathrm{QW}} n_{\downarrow}\left(I_{1}-I_{2}\right), \\
& \Delta E_{\downarrow}=2 n_{\downarrow}\left(I_{1}-I_{2}\right)+2 f_{\mathrm{QW}} n_{\uparrow}\left(I_{1}-I_{2}\right),
\end{aligned}
$$

where

$$
I_{1}=\int \frac{d \vec{q}}{(2 \pi)^{d}}\left|\phi_{0}(q)\right|^{3} \int \frac{d \vec{t}}{(2 \pi)^{d}} V(\vec{t}) \phi_{0}(\vec{q}+\vec{t})
$$

and

$$
I_{2}=\int \frac{d \vec{q}}{(2 \pi)^{d}}\left|\phi_{0}(\vec{q})\right|^{2} \int \frac{d \vec{t}}{(2 \pi)^{d}}\left|\phi_{0}(\vec{q}+\vec{t})\right|^{2} V(\vec{t}) .
$$

Since we are in the lowest order of perturbation theory $I_{1}$ and $I_{2}$ do not depend on the perturbed wave functions. In the Appendix we show that in 2D $I_{1}=\pi a^{2}\left|E_{0}^{2 \mathrm{D}}\right|$ and $I_{2}=\pi\left|E_{0}^{2 \mathrm{D}}\right| a^{2} 315 \pi^{2} / 2^{12}$, where $\left|E_{0}^{2 \mathrm{D}}\right|$ is the $2 \mathrm{D}$ Rydberg $E_{0}^{2 \mathrm{D}}=-2 e^{2} / \varepsilon a=-2 h^{2} / \varepsilon \mu a^{2}=4 E_{0}^{3 D}$. We obtain

$$
\begin{gathered}
\Delta E_{\uparrow}=k\left|E_{0}^{2 \mathrm{D}}\right| a^{2}\left(n_{\uparrow}+f_{\mathrm{QW}} n_{\downarrow}\right), \\
\Delta E_{\downarrow}=k\left|E_{0}^{2 \mathrm{D}}\right| a^{2}\left(n_{\downarrow}+f_{\mathrm{QW}} n_{\uparrow}\right), \\
\Delta^{2 \mathrm{D}} \equiv \Delta E_{\uparrow}-\Delta E_{\downarrow}=k\left|E_{0}^{2 \mathrm{D}}\right|\left(1-f_{\mathrm{QW}}\right)\left(n_{\uparrow}-n_{\downarrow}\right) a^{2},
\end{gathered}
$$

where $k=1.515$. We observe that the interexcitonic exchange interaction produces a blueshift of the levels. Although this calculation does not include screening effects, $\Delta^{2 \mathrm{D}}$ gives properly the spin splitting because screening is spin independent as discussed below. We observe that $\Delta^{2 \mathrm{D}}$ is proportional to the polarization $P=\left(n_{\uparrow}-n_{\downarrow}\right) /\left(n_{\uparrow}+n_{\downarrow}\right)$ and takes its maximum value when $f_{\mathrm{QW}}=0$, i.e., in the strictly $2 \mathrm{D}$ case. Anyway, $f_{\mathrm{QW}}$ is significantly smaller than 1 and we shall drop it in the following calculations. In the nonpolarized case we retrieve the result obtained by Schmitt-Rink et al. for energy shifts. ${ }^{31}$

In $3 \mathrm{D}$ we obtain analogously $I_{1}-I_{2}=13 \pi / 3$ which brings to

$$
\begin{aligned}
& \Delta E_{\uparrow}=13 \pi / 3\left|E_{0}^{3 \mathrm{D}}\right| a^{3}\left(\frac{10}{16} n_{\uparrow}+\frac{6}{16} n_{\downarrow}\right), \\
& \Delta E_{\downarrow}=13 \pi / 3\left|E_{0}^{3 \mathrm{D}}\right| a^{3}\left(\frac{10}{16} n_{\downarrow}+\frac{6}{16} n_{\uparrow}\right),
\end{aligned}
$$




$$
\begin{aligned}
\Delta^{3 \mathrm{D}} & \equiv \Delta E_{\uparrow}-\Delta E_{\downarrow}=13 \pi / 3\left|E_{0}^{3 \mathrm{D}}\right|\left(\frac{10}{16}-\frac{6}{16}\right)\left(n_{\uparrow}-n_{\downarrow}\right) a^{2} \\
& =3.4\left|E_{0}^{3 \mathrm{D}}\right|\left(n_{\uparrow}-n_{\downarrow}\right) a^{2} .
\end{aligned}
$$

The energy splitting between spin -1 and spin +1 excitons that occurs in the 3D case is, at equal densities $n a^{d}$, very close to the $2 \mathrm{D}$ one; both of them scaled in their corresponding Rydbergs $E_{0}^{d}$. Hence, we predict that the energy splitting, measured in units of $E_{0}^{\mathrm{QW}}$, has a very weak dependence on the quantum well width. On the other hand, in the 3D nonpolarized case we do not recover exactly the result obtained by Haug and Schmitt-Rink ${ }^{17}$ for energy shifts because we do not use spinless wave functions as they do. The effect of the spin part of the wave function is to set $A_{3 \mathrm{D}}=10 / 16$ instead of 1 as in their calculations.

We have been able to calculate the many-body corrections to the exciton binding energy in the case $n_{+2}=n_{-2}=0$ using perturbation theory. The many-body corrections in $E_{\uparrow}$ and $E_{\downarrow}$ do not depend on the energy levels of \pm 2 excitons. Therefore, we do not need to write the multiexciton equations for the $n_{+2} \neq n_{-2} \neq 0$ case. In order to avoid tedious algebra we can evaluate the energy corrections by counting how many integrals are nonzero in the sums of Eq. (6) noticing that each bracket makes a contribution equal to $I_{1}-I_{2}$ times the spin factor. In this way we obtain for the 2D case the following interexcitonic exchange corrections:

$$
\delta\left(\begin{array}{l}
E_{+2} \\
E_{+1} \\
E_{-1} \\
E_{-2}
\end{array}\right)=E_{0}^{2 \mathrm{D}} a^{2}\left(\begin{array}{cccc}
k & k & k & 0 \\
k & k & 0 & k \\
k & 0 & k & k \\
0 & k & k & k
\end{array}\right)\left(\begin{array}{c}
n_{+2} \\
n_{+1} \\
n_{-1} \\
n_{-2}
\end{array}\right) .
$$

A prediction of this theory is that, neglecting $f_{\mathrm{QW}}$-like factors, the $M$ excitons interact with equal strength with all the others except with the $(-M)$ excitons for which no interaction exists. The energy splitting of \pm 1 excitons is given by

$$
\Delta^{2 \mathrm{D}}=k\left|E_{0}^{2 \mathrm{D}}\right| a^{2}\left(n_{+1}-n_{-1}\right) .
$$

The \pm 2 exciton density is negligible compared with that of the \pm 1 excitons because the \pm 2 excitons cannot be optically generated in one photon processes. Therefore, their influence on $\Delta^{2 \mathrm{D}}$ is very small.

In the $3 \mathrm{D}$ case we have

$$
\delta\left(\begin{array}{l}
E_{+2} \\
E_{+1} \\
E_{-1} \\
E_{-2}
\end{array}\right)=\frac{13 \pi}{6} E_{0}^{3 \mathrm{D}} a^{3}\left(\begin{array}{cccc}
1 & 1 & \frac{1}{4} & 0 \\
1 & \frac{10}{16} & \frac{6}{16} & \frac{1}{4} \\
\frac{1}{4} & \frac{6}{16} & \frac{10}{16} & 1 \\
0 & \frac{1}{4} & 1 & 1
\end{array}\right)\left(\begin{array}{c}
n_{+2} \\
n_{+1} \\
n_{-1} \\
n_{-2}
\end{array}\right) .
$$

This equation does not carry new physics compared to Eq. (20) and, as in the 2D case, the influence of the \pm 2 excitons is limited because of their very low occupation. In a situation with $n_{ \pm 1} \geqslant 10^{10} \mathrm{~cm}^{-2}$ and \pm 2 exciton negligibly populated, the later energy levels are higher (less bound) than the \pm 1 excitons both in $2 \mathrm{D}$ and $3 \mathrm{D}$ because the \pm 2 excitons interact with two highly populated excitons, say, +1 and -1 .

\section{B. Screening corrections}

Hitherto we have used the unscreened Coulomb potential reduced by the dielectric constant $\epsilon$ of the material in its ground state. The presence of a considerable amount of excited mobile carriers (electrons and holes) screens the interaction between these carriers and the rest of the lattice. The renormalization of the exciton binding energy caused by screening has received considerable attention. ${ }^{16,20,32}$ In order to simplify the theory we have calculated the screening corrections in the random phase approximation (RPA) ${ }^{17}$ In this approximation the screening correction to the binding energy does not depend on the exciton spins ${ }^{17,32}$ being

$$
\Delta E_{0}^{s c} \simeq-n \sum_{\nu^{\prime}, \nu^{\prime \prime}, q} V^{2}(\vec{q}) \frac{\left.\left(\left|\left\langle 1 s\left|e^{i \alpha \vec{q} \cdot \vec{r}}-e^{-i \beta \vec{q} \cdot \vec{r}}\right| \nu^{\prime \prime}\right\rangle\right|\right)^{2}\left|\left\langle 1 s\left|e^{-i \alpha \vec{q} \cdot \vec{r}}-e^{i \beta \vec{q} \cdot \vec{r}}\right| \nu^{\prime}\right\rangle\right|\right)^{2}}{2\left(E_{0}+\frac{q^{2}}{2 M_{E X}}\right)},
$$

where $M_{E X} \equiv m_{e}+m_{h}$ is the total exciton mass and $|\nu\rangle$ are the exciton internal states, $\alpha \equiv m_{h} / M_{E X}$ and $\beta \equiv m_{e} / M_{E X}$. Using the completeness relation we get

$$
\begin{aligned}
\Delta E_{0}^{s c} & \simeq-2 n \sum_{\vec{q}} V^{2}(\vec{q}) \frac{[\langle 1 s|1-\cos (\vec{q} \cdot \vec{r})| 1 s\rangle]^{2}}{\left(E_{0}+\frac{q^{2}}{2 M_{E X}}\right)} \\
& =-2 n \sum_{\vec{q}} V^{2}(\vec{q})\left[1-\frac{1}{\left(1+a^{2} q^{2} / 4\right)^{2}}\right]^{2} \frac{1}{E_{0}+\frac{q^{2}}{2 M_{E X}}} .
\end{aligned}
$$

Next, we transform the summation in an integral following the usual prescription. The screening correction we obtain in the $2 \mathrm{D}$ case is

$$
\Delta E_{0}^{s c} \simeq-\pi n a^{2}\left|E_{0}^{2 \mathrm{D}}\right| w^{2} F(w),
$$

where $w \equiv(M / 4 \mu)^{1 / 2}$ and

$$
F(w)=\int_{0}^{\infty} \frac{d x}{x}\left[1-\frac{1}{\left(1+x^{2}\right)^{3 / 2}}\right]^{2} \frac{1}{x^{2}+w^{2}} .
$$


In a GaAs quantum well $z \equiv m_{h} / m_{e} \simeq 2.5$ which leads to $w^{2=(1+z)} / 4 z \simeq 1.2, \quad w^{2} F(w) \simeq 0.41, \quad$ and $\quad \Delta E_{0}^{s c} \simeq-$ $0.41 \pi n a^{2}\left|E_{0}^{2 \mathrm{D}}\right|$. The screening contribution reduces the effect of the bare Coulomb interaction between the carriers producing a relative redshift of all the exciton levels and therefore does not contribute to the spin level splitting. It must be stressed that $w^{2} F(w)$ is a very smooth function of $m_{h} / m_{e}$ and the screening correction is rather insensitive to variations of this mass ratio.

A physical interpretation of the way the screening modifies the exciton binding energy is the following. In the preceding section we calculated the exciton binding energies taking into account the interexcitonic interaction with the bare Coulomb potential $V_{0}$. Now we calculate the dressed Coulomb potential $V_{s}$ in the RPA and we treat the difference $V_{s}-V_{0}$ as a perturbation. It happens that, to the lowest order, $V_{s}-V_{0}$ is proportional ${ }^{17}$ to $n$ and we must use the noninteracting wave functions to evaluate the screening correction. Furthermore, we cannot calculate the screening corrections to the blueshifts caused by the interexcitonic exchange interaction because they would be, at least, of order $\left(n a^{2}\right)^{2}$. Hence the 2D exciton levels including interexcitonic exchange and screening are obtained from

$$
\begin{aligned}
\left(\begin{array}{l}
E_{+2} \\
E_{+1} \\
E_{-1} \\
E_{-2}
\end{array}\right) & =\left|E_{0}^{2 \mathrm{D}}\right|\left(\begin{array}{c}
-1 \\
-1 \\
-1 \\
-1
\end{array}\right) \\
& +\left|E_{0}^{2 \mathrm{D}}\right| a^{2}\left(\begin{array}{cccc}
k-q & k-q & k-q & -q \\
k-q & k-q & -q & k-q \\
k-q & -q & k-q & k-q \\
-q & k-q & k-q & k-q
\end{array}\right) \\
& \times\left(\begin{array}{l}
n_{+2} \\
n_{+1} \\
n_{-1} \\
n_{-2}
\end{array}\right)
\end{aligned}
$$

where the screening redshift constant $q=1.28$ is very close to the bare excitonic blueshift constant $k=1.515$ previously obtained.

The screening correction energy in bulk was calculated in RPA by Zimmermann ${ }^{32}$ obtaining

$$
\begin{aligned}
\Delta E_{0}^{s c} \simeq & \pi\left|E_{0}^{3 \mathrm{D}}\right| n a^{3}\left(\frac{w\left(32+63 w+44 w^{2}+11 w^{3}\right)}{(1+w)^{4}}\right. \\
& \left.-\frac{8 w(4+3 w)}{(1+w)^{2}}\right) \equiv \pi\left|E_{0}\right| n a^{3} f(w) .
\end{aligned}
$$

As in the $2 \mathrm{D}$ case, the screening correction in the RPA is quite insensitive to variations of $z$. Hence, we also take $z \equiv m_{h} / m_{e} \simeq 2.5$ for $3 \mathrm{D} \mathrm{GaAs}$ which leads $f[w]=-5.0$ and $\Delta E_{0}^{s c} \simeq-5 \pi\left|E_{0}^{3 \mathrm{D}}\right| n a^{3}$. Hence, the bulk interacting exciton levels are given by Eq. (23) minus the screening correction $\Delta E_{0}^{s c}$.

\section{Variational approach}

We have a set of complicated equations [(13) and (14)] for which we have applied first order perturbation theory. We cannot go beyond first order due to our previous hypothesis but we would like to extract more information from those equations. In order to do that we have tried a simple variational approach in the $2 \mathrm{D}$ case. As we did in the perturbation approach we treat Eqs. (13) and (14) as a Schrödinger equation. We identify a Hamiltonian and minimize $\langle\phi(q, \alpha)|H| \phi(q, \alpha)\rangle$, with

$$
\phi_{0}^{\mathrm{var}}(q)=\frac{(2 \pi)^{1 / 2} a \alpha}{\left[1+(a \alpha q / 2)^{2}\right]^{3 / 2}}
$$

i.e., we use the exciton radius as a variational parameter. This ansatz may be improved if we make $\alpha$ dependent on the spin. However, the calculations are much simpler with ansatz 30. The nontrivial integrals we have to perform are precisely $I_{1}$ and $I_{2}$ scaling $a$ with the variational parameter $\alpha$. After some algebra we arrive at

$$
\begin{aligned}
E_{M}\left(\alpha, n, n_{M}\right)= & \left|E_{0}^{2 \mathrm{D}}\right|\left[\frac{1}{(\alpha)^{2}}-\frac{2}{\alpha}+1.515 n_{M} a^{2} \alpha\right. \\
& \left.-1.28 n a^{2}(\alpha)^{2}\right],
\end{aligned}
$$

where $n$ is the total density and $n_{M}$ is the $M$-exciton density. In this expression we have set $f=0$. Now we have to look for the $\alpha$ that minimizes $E_{M}\left(\alpha, n, n_{M}\right)$ for each $\mathbf{n}$. We have done that numerically obtaining that, up to $n a^{2} \simeq 0.2$, the variational technique and the perturbation theory predict the same energy $\Delta E(M, \mathbf{n})$ with an error less than $1 \%$ and $\alpha$ does not differ from 1 (the perturbation theory value) more than a few percent. We can conclude that, in the small density limit $\left(n a^{2} \leqslant 0.2\right)$, the energy is properly given by first order perturbation theory and the wave function is the independent exciton one.

\section{EXCITON SPIN DYNAMICS}

One could expect our theory to predict new spin flip channels originated by the interaction. However, this is not the case because the interaction terms in Eqs. (13) and (14) are proportional to $n$ and the transitions rates are proportional to the squared interactions terms, i.e., $n^{2}$. Hence, following the considerations made in Sec. II, we must neglect these "interacting" transition rates: our theory predicts no significant variations of transitions rates with respect to those of the noninteracting theory ${ }^{7}$ while the energy levels correspond to Eq. (28). Therefore, we borrow the population evolution equation from Ref. 7 ,

$$
\frac{d \mathbf{n}}{d t}=\mathcal{W} \mathbf{n}
$$

where 


$$
\mathcal{W} \equiv\left(\begin{array}{cc}
-\left(W_{2,1}^{e}+W_{2,-1}^{h}\right) & W_{1,2}^{e} \\
W_{2,1}^{e} & -\left(W_{R}+W_{e x}^{1,-1}\right. \\
& \left.+W_{1,2}^{e}+W_{1,-2}^{h}\right) \\
W_{2,-1}^{h} & W_{e x}^{1,-1} \\
& \\
0 & W_{1,-2}^{h}
\end{array}\right.
$$

This equation is obtained from the master equation by making the approximation of quasi-equilibrium and taking into account the detailed balance principle. ${ }^{33}$ There are three kinds of transition rates: the easiest to understand is the radiative recombination rates $W_{R}$ which affect only the optically active excitons \pm 1 . Also, we have the $W_{M, M^{\prime}}^{e(h)}$ rates, say, the transition $(M)$ exciton to $\left(M^{\prime}\right)$ exciton caused by the $e(h)$ spin flip. The last type of transition rate, $W_{e x}^{M, M^{\prime}}$, is associated to the intraexcitonic exchange mechanism. Following Ref. 7 we set

$$
\begin{gathered}
W_{R}=1 / 400 \mathrm{ps}, \\
W_{M, M^{\prime}}^{e(h)}=\frac{1}{\tau^{e(h)}} \frac{1}{1+\exp \left[\left(E_{M^{\prime}}-E_{M}\right) \beta\right]}, \\
W_{e x}^{M, M^{\prime}}=\frac{1}{\tau^{e x}} \frac{1}{1+\exp \left[\left(E_{M^{\prime}}-E_{M}\right) \beta\right]},
\end{gathered}
$$

where $\tau^{e, h}$ are the single particle spin flip times, ${ }^{7,8,13} \tau^{e x}$ is the exchange spin flip time calculated by Maialle et al., ${ }^{7}$ $\beta=1 / k_{B} T$, and $E_{M}$ are those of Eq. (28). The numerical values of $\tau^{e(h)}$ and $\tau^{e x}$ are taken from the case II of Ref. 7 .

\section{RESULTS AND CONCLUSIONS}

The solution of the nonlinear equations (32) is obtained numerically by a Runge-Kutta method. Inserting the time dependence of the densities in Eq. (28) we obtain the time evolution of the exciton levels. In our theoretical calculations the natural energy scale is the Rydberg (the $2 \mathrm{D}$ or $3 \mathrm{D}$ ). In order to compare our results with experiments ${ }^{2-6}$ we shall plot the energies in units of $E_{0}^{\mathrm{QW}}$. We have performed calculations with different values for $f_{\mathrm{QW}}$ that, as discussed in Sec. II, must be greater than 0 (2D value) and smaller than $3 / 8$ (3D value). The results do not depend qualitatively on the particular value for $f_{\mathrm{QW}}$ and we present here results for $f_{\mathrm{QW}}=3 / 80$, i.e., close to the $2 \mathrm{D}$ value, because we want to compare with experiments in very narrow wells. ${ }^{34}$ On the other hand, we have checked that the results are insensitive to the variation of the initial populations of \pm 2 excitons provided they are less than $10 \%$ of the total initial density $n_{0}$ and, consequently, we present figures obtained from an initial density of \pm 2 excitons equal to zero. We adopt the following conventions: (i) the $(+1)$ exciton is the more populated state at $t=0$; (ii) the populations are measured in $n_{0}$ units; (iii) the origin of energies is taken at the bottom of the conduction band.

In Fig. 1 we plot our theoretical predictions of populations

$$
\left.\begin{array}{cc}
W_{-1,2}^{h} & 0 \\
W_{e x}^{-1,1} & W_{-2,1}^{h} \\
-\left(W_{R}+W_{e x}^{-1,1}\right. & W_{-2,-1}^{e} \\
\left.+W_{-1,-2}^{e}+W_{-1,2}^{h}\right) & \\
W_{-1,-2}^{e} & -\left(W_{-2,-2}^{e}+W_{-2,-1}^{h}\right)
\end{array}\right) .
$$

(a) and energy levels (b) with an initial density of $n a^{2}=0.1$ (about $10^{11}$ excitons per $\mathrm{cm}^{2}$ ) and an initial polarization $P=\left(n_{\uparrow}-n_{\downarrow}\right) /\left(n_{\uparrow}+n_{\downarrow}\right)=80 \%$. In Fig. 1(a) we observe that the polarization disappears in roughly $50 \mathrm{ps}$. As we see in the inset the \pm 2 populations are, at least, one order of magnitude smaller and they decay much slower than the optically active ones. In Fig. 1(b) the more important features are the splitting between spin +1 and spin -1 excitons and the fact that the \pm 2 excitons levels are closer to the conduction band than the \pm 1 ones. In $t=0$ the splitting takes its maximum value, $0.25 E_{0}^{\mathrm{QW}}$, about $2 \mathrm{meV}$, and then decreases becoming zero at $t=50 \mathrm{ps,}$ as observed experimentally. ${ }^{5}$ It must be stressed that the contrapolarized (less populated) exciton level shows an energy redshift and
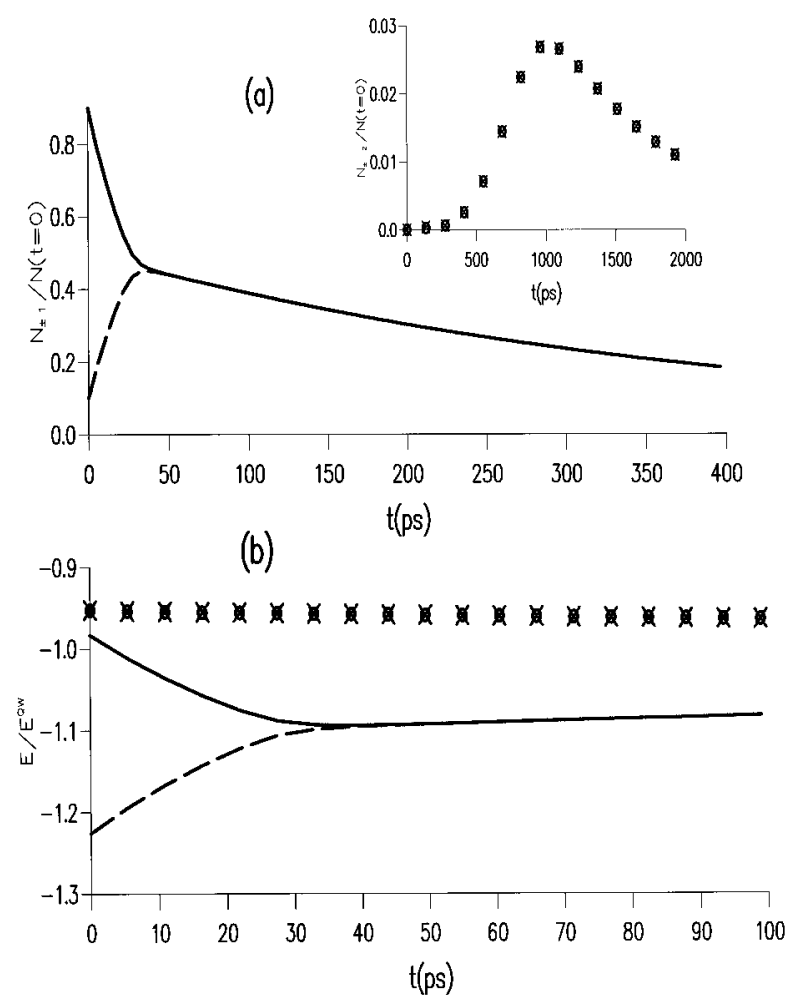

FIG. 1. (a) \pm 1 exciton population as a function of time $(0-400$ ps). The dashed line is the -1 population. In the inset we plot the +2 (circles) and -2 (crosses) populations (notice the different time scale). (b) Exciton levels as a function of time (0-140 ps) measured in quantum well free exciton energy (see text). Crosses and circles are again the \pm 2 excitons. The dashed line corresponds to $(-1)$ exciton energy. Initial density $n a^{2}=0.1$, initial polarization $P=80 \%$. 

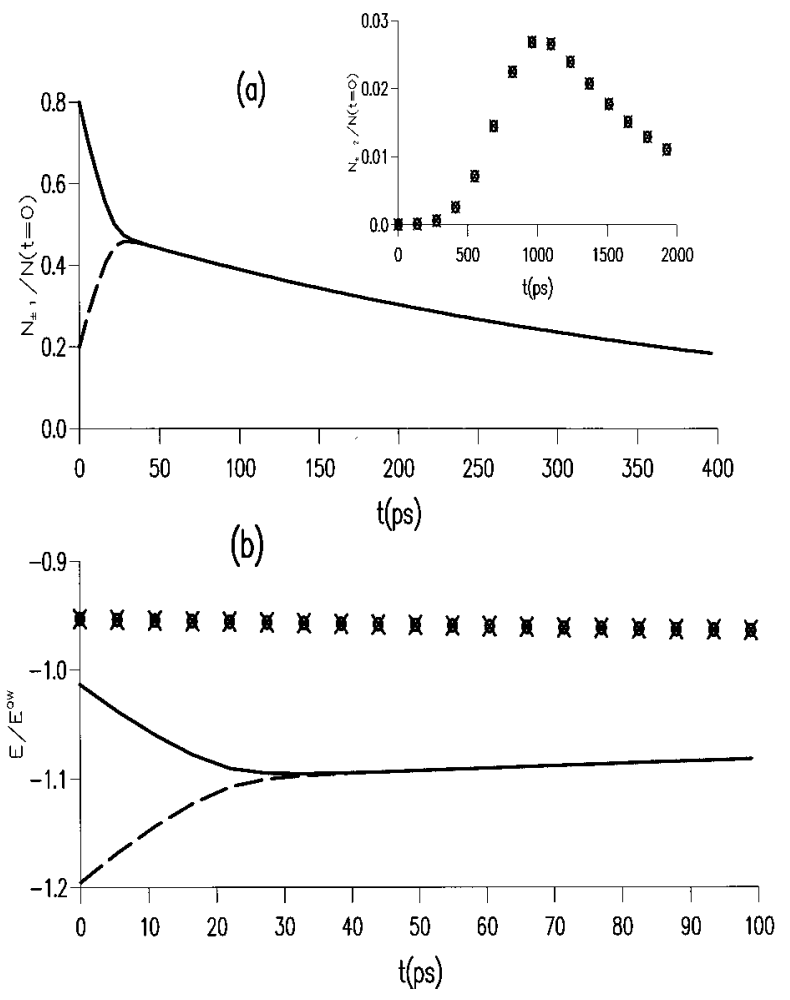

FIG. 2. As in Fig. 1 with $n=0.1, P=60 \%$.

the copolarized (more populated) starts having a blueshift and, when its population decreases, presents a redshift. We also show similar results with either different initial polarization (Fig. 2) or population (Fig. 3). The main difference between Fig. 2 where $P=60 \%$ and Fig. 1 where $P=80 \%$ is the decrease of the \pm 1 splitting as well as a shorter duration of this splitting. In Fig. 3 we set the initial population $n a^{2}$ equal to 0.05 (about $5.0 \times 10^{10}$ excitons per $\mathrm{cm}^{2}$ ) and the initial polarization once again $P=80 \%$. The splitting is smaller than the one observed in Fig. 1, about $0.25 E_{0}^{\mathrm{QW}}$ and its duration is longer.

The main consequences we extract from these results are the following.

(i) The spin level degeneracy breaking is proportional to the polarization of excitons populations and both polarization and splitting disappear in a time of the order of 50 ps.

(ii) The \pm 2 excitons are negligibly populated and therefore do not influence the \pm 1 exciton energy levels, although the opposite is true: the \pm 1 excitons do influence the \pm 2 energy levels.

In order to compare with experimental information, an interesting way of presenting our results is to give energy levels at fixed times as a function of the initial exciton density. In Fig. 4 some of the conclusions we stated before become more clear. The splitting increases with $P$ and $n$. Some experiments ${ }^{34}$ seem to suggest that the majority carrier level remains essentially constant as a function of the initial density, something that happens in our results, where the variation of this energy with $n$ is very small for the majority excitons. This is a consequence of the cancellation between the exchange $(k)$ and screening $(q)$ corrections mentioned in Sec. III B. Our theory gives good results for level splittings

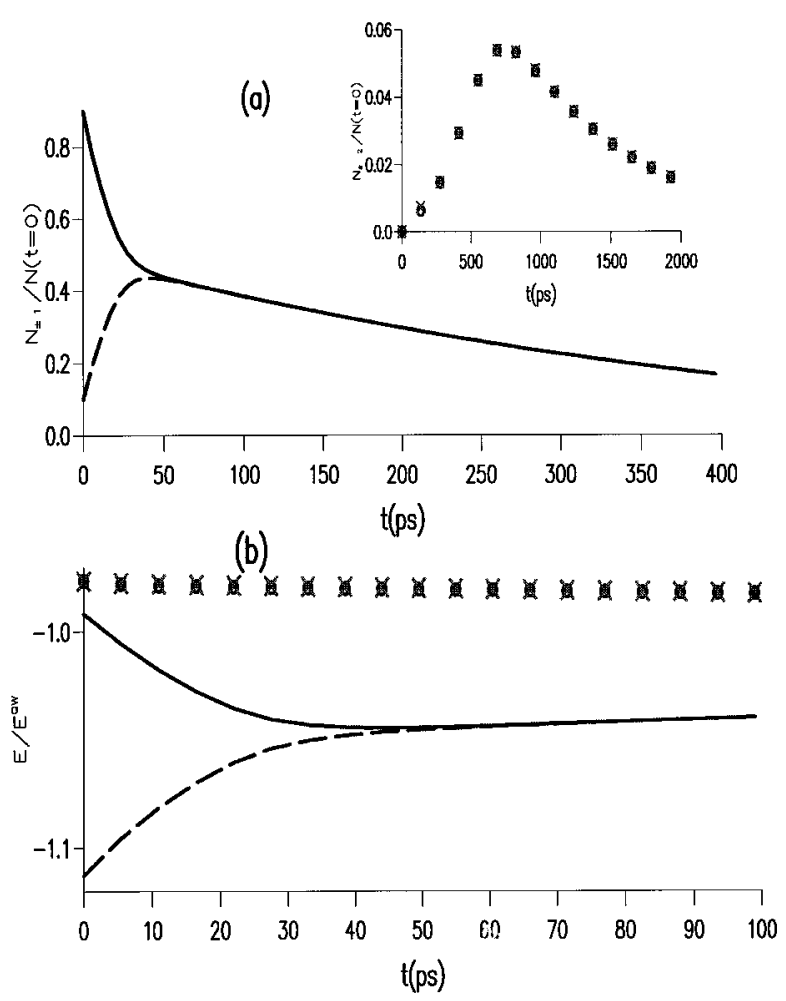

FIG. 3. As in Fig. 1 with $n=0.05, P=80 \%$.

but fails to describe in detail the absolute position of the excitonic luminescence peaks. ${ }^{2,3,34}$ These disagreements can be due to effects not taken into account in our theory: (i) Mixing effects in the valence band connected with finite width of actual quantum wells in the growth direction; ${ }^{35}$ (ii) pinning of excitons to impurities, an effect that might change the exciton energies; and (iii) failure of the approximation of considering the center of mass at rest to explain nonresonant excitation experiments.

In summary, we have presented a theory which describes the interacting polarized exciton gas with moderately high density. We have obtained a set of multiexciton Wannier equations that we have solved, both perturbativately and variationally, obtaining energy shifts and splittings originated by the interexcitonic exchange interaction. Whenever differences between the exciton populations exist, energy levels are split. Calculated and experimentally observed splittings are in good qualitative agreement.

\section{ACKNOWLEDGMENTS}

This work was supported in part by the Comisión Interministerial de Ciencia y Tecnologí a of Spain under Contract No. MAT 94-0982-C02-01, by the Comunidad Autónoma de Madrid under Contract No. AE00330/95, and by the Commission of European Communities under Contract Ultrafast CHRX-CT93-0133.

\section{APPENDIX: CALCULATION OF $I_{1}$ AND $I_{2}$}

In this appendix we calculate $I_{1}$ and $I_{2}$ both in $2 \mathrm{D}$ and 3D. The calculation of 

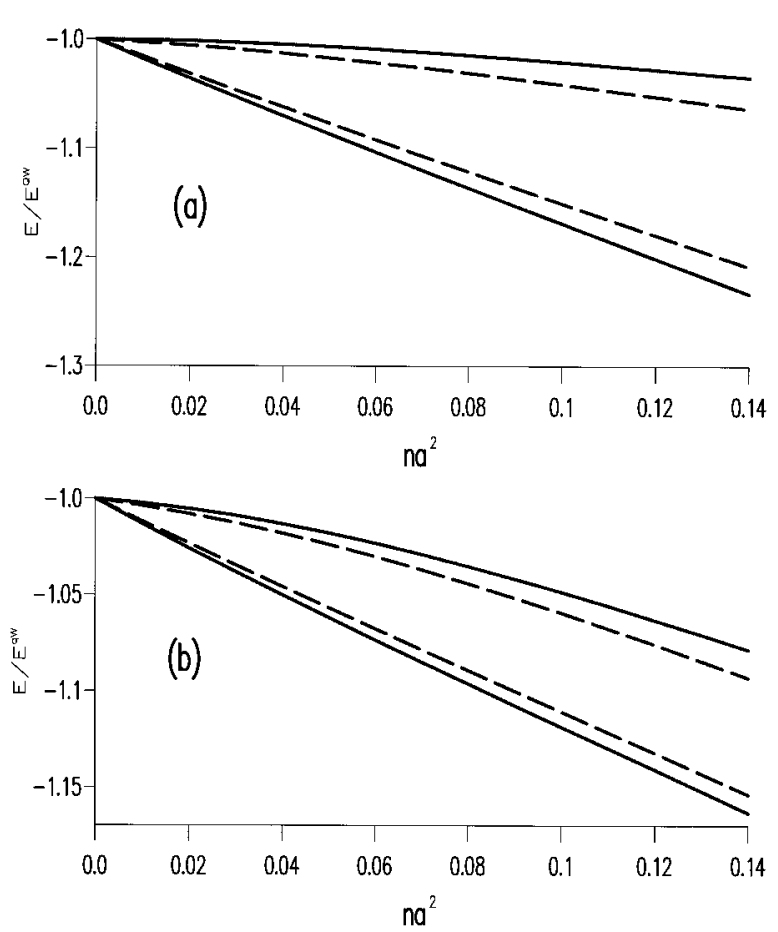

FIG. 4. \pm 1 exciton energies at (a) $t=10 \mathrm{ps}$ and (b) $t=25 \mathrm{ps}$ as a function of initial densities. The solid and dashed lines correspond to an initial polarization of $80 \%$ and $60 \%$, respectively, and upper and lower lines correspond to the spin +1 and spin -1 exciton, respectively. In order to compare with experiments one must take into account that $a$ is roughly $100 \AA$.

$$
I_{1}=\int \frac{d \vec{q}}{(2 \pi)^{d}}\left|\phi_{0}(q)\right|^{3} \int \frac{d \vec{t}}{(2 \pi)^{d}} V(\vec{t}) \phi_{0}(\vec{q}+\vec{t})
$$

can be done both in $2 \mathrm{D}$ and $3 \mathrm{D}$ by using the Schrödinger equation

$$
\int \frac{d \vec{t}}{(2 \pi)^{d}} V(\vec{t}) \phi_{0}(\vec{q}+\vec{t})=\left[\frac{q^{2}}{2 \mu}-E_{0}\right] \phi_{0}(q) .
$$

Substituting (A2) in (A1) we obtain

$$
I_{1}=\int \frac{d \vec{q}}{(2 \pi)^{d}}\left|\phi_{0}(q)\right|^{4}\left[\frac{q^{2}}{2 \mu}-E_{0}\right] .
$$

In the $2 \mathrm{D}$ case we obtain for this integral

$$
I_{1}=8 \pi a^{2}\left|E_{0}^{2 \mathrm{D}}\right| \int_{0}^{\infty} \frac{x d x}{\left(1+x^{2}\right)^{5}}=\pi\left|E_{0}^{2 \mathrm{D}}\right| a^{2}
$$

and in the $3 \mathrm{D}$ case

$$
I_{1}=2^{11}\left|E_{0}^{3 \mathrm{D}}\right| a^{3} \int_{0}^{\infty} \frac{x^{2} d x}{\left(1+x^{2}\right)^{7}}=21 \pi\left|E_{0}^{3 \mathrm{D}}\right| a^{3} .
$$

The calculation of

$$
I_{2}=\int \frac{d \vec{q}}{(2 \pi)^{d}}\left|\phi_{0}(\vec{q})\right|^{2} \int \frac{d \vec{t}}{(2 \pi)^{d}}\left|\phi_{0}(\vec{q}+\vec{t})\right|^{2} V(\vec{t})
$$

is more cumbersome. We start with the 2D case by perfoming the integral

$$
\begin{aligned}
\Sigma_{00} & \equiv \int \frac{d \vec{t}}{(2 \pi)^{2}}\left|\phi_{0}(\vec{q}+\vec{t})\right|^{2} V(\vec{t}) \\
& =\int \frac{d \vec{s}}{(2 \pi)^{2}} V(\vec{s}) \int d \vec{r} \chi(\vec{r}) e^{-i(\vec{s}+\vec{q}) \cdot \vec{r}} \\
& =\int d \vec{r} \chi(\vec{r}) e^{-i \vec{q} \cdot \vec{r}} \int \frac{d \vec{s}}{(2 \pi)^{2}} V(\vec{s}) e^{-i \vec{s} \cdot \vec{r}}
\end{aligned}
$$

where

$$
\begin{aligned}
\chi(\vec{r}) & \equiv \int \frac{d \vec{t}}{(2 \pi)^{2}}\left|\phi_{0}(\vec{t})\right|^{2} e^{i \vec{t} \cdot \vec{r}}=\frac{1}{2 \pi} \int_{0}^{\infty} t J_{0}(t r)\left|\phi_{0}(t)\right|^{2} \\
& =a^{2} \int_{0}^{\infty} \frac{t J_{0}(t r) d t}{\left.[1+a t / 2)^{2}\right]^{3}}=\frac{\gamma^{2}}{2} K_{2}(\gamma)
\end{aligned}
$$

where $J_{0}(x)$ is the Bessel function of the first kind, $K_{2}(\gamma)$ is the modified Bessel function, and $\gamma \equiv 2 r / a$. Since $V(\vec{s})$ is the Fourier-transform of the Coulomb potential,

$$
\int \frac{d \vec{s}}{(2 \pi)^{2}} V(\vec{s}) e^{-i \vec{s} \cdot \vec{r}}=\frac{e^{2}}{r}
$$

and we obtain

$$
\begin{aligned}
\Sigma_{00}= & \frac{4 \pi e^{2}}{a^{2}} \int_{0}^{\infty} r^{2} K_{2}\left(\frac{2 r}{a}\right) J_{0}(q r) d r=3 \pi^{2} e^{2} a_{2} F_{1}\left[\frac{1}{2}, \frac{5}{2}, 1\right. \\
& \left.-(q a / 2)^{2}\right]
\end{aligned}
$$

where

$$
{ }_{2} F_{1}[a, b, c, x] \equiv \sum_{k=0}^{\infty} \frac{(a)_{k}(b)_{k}}{(c)_{k}} \frac{x^{k}}{k !} ; \quad\left(a_{k}\right) \equiv \frac{(a+k-1) !}{(a-1) !}
$$

is the confluent hypergeometrical function. In order to obtain $I_{2}$ we use (A10) in (A6) and we get

$$
I_{2}=3 \pi^{2} a e^{2} \int_{0}^{\infty} \frac{x_{2} F_{1}\left[\frac{1}{2}, \frac{5}{2}, 1,-x^{2}\right] d x}{\left(1+x^{2}\right)^{3}}=\frac{315}{4096} \pi^{3}\left|E_{0}^{2 \mathrm{D}}\right| a^{2},
$$

where we have used

$$
\int_{0}^{\infty} \frac{x_{2} F_{1}\left[\frac{1}{2}, \frac{5}{2}, 1,-x^{2}\right] d x}{\left(1+x^{2}\right)^{3}}=\frac{2}{3} \pi \frac{315}{4096} .
$$

In $3 \mathrm{D}$ the calculation of $I_{2}$ is easier. We have

$$
\begin{aligned}
I_{2} & =\int \frac{d \vec{q}}{(2 \pi)^{d}}\left|\phi_{0}(\vec{q})\right|^{2} \int \frac{d \vec{t}}{(2 \pi)^{d}}\left|\phi_{0}(\vec{q}+\vec{t})\right|^{2} V(\vec{t}) \\
& =\frac{2^{11}}{\pi} e^{2} a^{2} \int_{0}^{\infty} \frac{x^{2} d x}{\left(1+x^{2}\right)^{4}} \int_{-1}^{+1} d \xi \int_{0}^{\infty} \frac{d y}{\left(1+x^{2}+y^{2}-2 x y \xi\right)^{4}},
\end{aligned}
$$

where $x \equiv a|\vec{q}|, y \equiv a|\vec{t}|$, and $a^{2} \vec{q} \vec{t}=x y \cos (\xi)$. Now we perform the integrations over $\xi$ and $y$ in two steps, 


$$
\begin{aligned}
F(x, y) \equiv & \int_{-1}^{+1} \frac{d \xi}{\left(1+x^{2}+y^{2}-2 x y \xi\right)^{4}} \\
= & \frac{1}{6 x y}\left\{\frac{1}{\left(1+x^{2}+y^{2}-2 x y\right)^{3}}\right. \\
& \left.-\frac{1}{\left(1+x^{2}+y^{2}+2 x y\right)^{3}}\right\}
\end{aligned}
$$

and

$$
\int_{0}^{\infty} \frac{d y}{y} F(x, y)=\frac{\pi}{8} x \frac{15+10 x^{2}+3 x^{4}}{\left(1+x^{2}\right)^{3}} \equiv J(x) .
$$

Hence, we obtain

$$
I_{2}=\frac{2^{12}}{6 \pi}\left|E_{0}^{3 \mathrm{D}}\right| a^{3} \int_{0}^{\infty} \frac{x J(x) d x}{\left[1+x^{2}\right]^{4}}=\frac{100}{6} \pi\left|E_{0}^{3 \mathrm{D}}\right| a^{3} .
$$

${ }^{1}$ Following the habitude in the field, we denote the third component of the exciton angular moment with the word spin.

${ }^{2}$ T.C. Damen, L. Viña, J.E. Cunningham, J. Shah, and L.J. Sham, Phys. Rev. Lett. 67, 3432 (1991).

${ }^{3}$ L. Muñoz, E. Pérez, L. Vĩna, J. Fernández-Rossier, C. Tejedor, and K. Ploog, Solid State Electron. 40, 755 (1996).

${ }^{4}$ B. Baylac, T. Amand, M. Brousseau, X. Marie, B. Dareys, G. Bacquet, J. Barrau, and R. Planel, Semicond. Sci. Technol. 10, 295 (1995).

${ }^{5}$ T. Amand, X. Marie, B. Baylac, B. Dareys, J. Barrau, M. Brousseau, R. Planel, and D.J. Dunstan, Phys. Lett. A 193, 105 (1995).

${ }^{6}$ J.B. Stark, W.H. Knox, and D.S. Chemla, Phys. Rev. B 46, 7919 (1992).

${ }^{7}$ M.Z. Maialle, E.A. de Andrada e Silva, and L.J. Sham, Phys. Rev. B 47, 15776 (1993).

${ }^{8}$ G.L. Bir, A.G. Aronov, and G.E. Pikus, Sov. Phys. JETP 42, 705 (1975).

${ }^{9}$ For an excellent review on noninteracting excitons see M.M. Denisov and V.P. Makarov, Phys. Status Solidi. B 56, 9 (1973).

${ }^{10}$ R. Knox, Solid State Physics, Suppl. 5 (Academic Press, New York, 1963).

${ }^{11}$ G.E. Pikus and G.L. Bir, Sov. Phys. JETP 33, 108 (1971).

${ }^{12}$ V.A. Kiselev and A.G. Zhilich, Sov. Phys. Solid State 13, 2008 (1972).

${ }^{13}$ M.I. D'Yakonov and V.I. Perel, Sov. Phys. JETP 33, 1053 (1971).

${ }^{14}$ R.J. Elliot, Phys. Rev. 96, 266 (1954); Y. Yafet, Solid State Physics (Academic Press, New York, 1963), Vol. 14, p. 1.

${ }^{15}$ E. Hanamura and H. Haug, Phys. Rep., 33C, 209 (1977).

${ }^{16}$ C. Comte and P. Nozieres, J. Phys. 43, 1069 (1982); 43, 1082 (1982).

${ }^{17}$ H. Haug and S. Schmitt-Rink, Prog. Quantum Electron. 9, 3 (1984)

${ }^{18}$ L.V. Keldysch and N. Kozlov, Zh. Éksp. Teor. Fiz. 54, 978 (1968) [Sov. Phys. JETP 27, 521 (1968)].
${ }^{19}$ R. Zimmermann, K. Kilimann, W.D. Kraeft, D. Kremp, and G. Röpke, Phys. Status Solidi B 90, 175 (1978); G. Röpke, T. Seifert, H. Stoltz, and R. Zimmermann, ibid. 100, 215 (1980).

${ }^{20}$ W.D. Kraeft, D. Kremp, W. Ebeling, and G. Röpke, Quantum Statistics of Charged Particle Systems (Akademie-Verlag, Berlin, 1986).

${ }^{21}$ L.J. Sham and T.M. Rice, Phys. Rev. 144, 708 (1966).

${ }^{22}$ V.P. Makarov, Sov. Phys. JETP 27, 173 (1968).

${ }^{23}$ G.Mahan, Many Particle Physics (Plenum Press, New York, 1982).

${ }^{24}$ H. Stoltz, R. Zimmermann, and G. Röpke, Phys. Status Solidi B 105, 585 (1981).

${ }^{25}$ R. Jin, K. Okada, G. Khitrova, H.M. Gibbs, M. Pereira, S.W. Koch, and N. Peyghambarian, Appl. Phys. Lett. 61, 1745 (1982).

${ }^{26}$ M.F. Pererira, Jr., R. Binder, and S.W. Koch, Appl. Phys. Lett. 63, 1201 (1994).

${ }^{27}$ G. Bastard, E.E. Mendez, L.L. Chang, and L. Esaki, Phys. Rev. B 26, 1974 (1982); G. Bastard, Wave Mechanics Applied to Semiconductor Heterosturctures (JOUVE, Paris, 1988).

${ }^{28}$ R.L. Greene, K.K. Bajaj, and D.E. Phelps, Phys. Rev. B 29, 1807 (1984).

${ }^{29}$ F. Bassani and G.P. Parravicini, Electronic States and Optical Transitions in Solids (Pergamon Press, London, 1975).

${ }^{30}$ C. Cohen-Tannoudji, B. Diu, and F. Laloë, Quantum Mechanics (John Wiley \& Sons, New York, 1977).

${ }^{31}$ S. Schmitt-Rink, D.S. Chemla, and A.B. Miller, Phys. Rev. B 32, 6601 (1985); Adv. Phys. 38, 89 (1989).

${ }^{32}$ R. Zimmermann, Phys. Status Solidi B, 76, 191 (1976).

${ }^{33}$ P. Van Kampen, Stochastic Processes in Physics and Chemistry (North-Holland, Amsterdam, 1992).

${ }^{34}$ L. Muñoz, E. Pérez, L. Viña, J. Fernández-Rossier, C. Tejedor, and K. Ploog (unpublished).

${ }^{35}$ S.H. Park, J.F. Morhange, A.D. Jeffery, R.A. Morgan, A. ChavezPirson, H.M. Gibbs, S.W. Koch, N. Peyghambarian, M. Derstine, A.C. Gossard, J.H. English, and W. Weigmann, Appl. Phys. Lett. 52, 1201 (1988). 